\title{
Le VEGF-C et son récepteur FIt4/VEGFR-3 : des vaisseaux lymphatiques aux vaisseaux sanguins
}

Dans la famille du VEGF (vascular endothelial growth factor), le VEGF-C et son récepteur VEGFR-3 ou Flt-4 occupaient une place à part, parce qu'ils semblaient jouer un rôle relativement spécifique dans le contrôle de la formation du système des vaisseaux lymphatiques. Le VEGFR-3 ne fixe pas le VEGF. Seules les formes clivées du VEGF-C se fixent à la fois sur les VEGFR-2 et -3 .

Les premiers travaux d'hybridation in situ révélaient une expression du gène codant pour le VEGFR-3 dans l'endothélium des veinules et des vaisseaux lymphatiques au cours du développement embryonnaire, tandis que les ARNm codant pour son ligand, le VEGF-C, étaient détectés essentiellement dans les régions où ces vaisseaux lymphatiques se forment par bourgeonnement à partir des veines de l'embryon [1]. In vitro, le VEGF-C stimule peu efficacement la prolifération des cellules endothéliales $(0,01 \mathrm{ng} \mathrm{VEGF}$ ont le même effet que $10 \mathrm{ng}$ VEGF-C). Sur la membrane chorioallantoïde de poulet, le VEGF et le VEGF-C induisent respectivement la formation des vaisseaux sanguins et celle des vaisseaux lymphatiques [2]. Enfin, les souris transgéniques chez lesquelles l'ADNc codant pour le VEGF-C avait été placé en aval du promoteur du gène de la kératine 14 présentent une hyperplasie des vaisseaux lymphatiques, sans que les artères et les veines soient affectées [3].

L'inactivation du gène codant pour le VEGFR-3 entraîne un tableau plus complexe : elle montre que ce récepteur joue un rôle dans la formation des vaisseaux sanguins, bien avant l'émergence des vaisseaux lymphatiques [4]. Cette inactivation a été obtenue en insérant le gène codant pour la $\beta$-galactosidase dans le premier exon du gène VEGFR-3. L'expression de VEGFR-3 ne commence qu'au cours du jour embryonnaire 8 (E8), et à E9, les embryons VEGFR-3-1- ont le même aspect général que les embryons sauvages. Mais, sur des coupes histologiques, les grands vaisseaux comme l'aorte ou la veine cardinale inférieure présentent des contours irréguliers et des lumières très rétrécies. Chez ces embryons à E10, le plexus vasculaire qui entoure la tête est constitué de capillaires de taille homogène, alors qu'il forme chez les embryons sauvages ou hétérozygotes un arbre hiérarchisé avec des petits et des gros vaisseaux. A E12, les embryons VEGFR-3-1- présentent de nombreuses zones de nécrose et à E14 il n'en reste quasiment pas de vivant : la défaillance cardiaque est majeure dès $\mathrm{E} 9,5$ et du liquide s'accumule dans la cavité péricardique. Les hétérozygotes sont normaux; ils ont permis d'analyser l'expression du gène grâce au marqueur $\beta$-galactosidase. A la naissance, les gros vaisseaux lymphatiques sont seuls marqués, mais, au cours du développement, le gène VEGFR-3 s'exprime d'abord dans les vaisseaux sanguins et semble indispensable au remodelage des premiers capillaires en vrais vaisseaux aux tailles hiérarchisées.

Chacun des membres de la famille des récepteurs du VEGF joue donc un rôle particulier dans la formation des vaisseaux sanguins [5]. VEGFR-2 (Flk-1/KDR) agit le premier. Il est essentiel pour la différenciation des cellules endothéliales et des cellules hématopoiétiques. Il serait impliqué dans la migration des hémangio- blastes, précurseurs présomptifs des deux lignages, vers le sac vitellin $(\mathrm{m} / \mathrm{s}$ 1998, $n^{\circ}$ 5, p. 662). Le phénotype des embryons VEGFR-1(Flt-1) $)^{-/}$suggère que ce récepteur contrôlerait la prolifération des cellules endothéliales dans l'embryon. En son absence, les parois des vaisseaux sont élargies, et des groupes de cellules endothéliales sont présents dans la lumière de ces vaisseaux. Il est intéressant de noter que la délétion du domaine tyrosine kinase du VEGFR-1 n'affecte pas le développement des souris [6]. On peut considérer que ce récepteur se contente de fixer le VEGF, sans stimuler en aval les voies de signalisation. Il est aussi possible que la signalisation par le VEGFR-1 ne nécessite pas l'activité tyrosine kinase de ce récepteur. Le VEGFR-3 agirait donc plus tard pour contrôler l'organisation de l'arbre vasculaire. Cette brève revue ne serait pas complète s'il n'était pas rappelé que la neuropiline-1, un nouveau récepteur de l'isoforme $\mathrm{VEGF}_{165}$, avait d'abord été observée dans les cellules nerveuses où elle dirigeait la trajectoire des nerfs périphériques $\left(\mathrm{m} / \mathrm{s} 1998, n^{\circ} 6-7\right.$, p. 811). Mais la mort in utero des embryons neuropiline- $1^{-1-}$ serait due à des déficiences de leur système cardio-vasculaire.

La saga du VEGF et de ses récepteurs illustre combien la lecture des articles qui relatent le phénotype des embryons ayant subi une invalidation génique requiert rigueur et prudence. Pour communiquer plus facilement, les auteurs ont tendance à retenir dans un phénotype complexe les points qui leur semblent les plus remarquables, en laissant de coté des aspects dont l'importance peut apparaître ultérieurement. En outre, la 
validité des conclusions est parfois limitée à la souche de souris utilisée. Enfin, plusieurs indications suggèrent que les mécanismes qui contrôlent la formation des vaisseaux sanguins ne sont pas les mêmes chez l'embryon et chez l'adulte [7].

B.V.

1. Kukk E, Lymboussaki A, Taira S, Kaipainen A Jeltsch M, Joukov V, Alitalo K. VEGF-C receptor binding and pattern of expression with VEGFR-3 suggests a role in lymphatic vascular development. Development 1996; 122: 3829-37.

2. Oh SJ, Jeltsch MM, Birkenhager R, McCarthy JE, Weich HA, Christ B, Alitalo K, Wilting J. VEGF and VEGF-C: specific induction of angiogenesis and lymphangiogenesis in the differentiated avian chorioallantoic membrane. Dev Biol 1997; 188: 96-109. 3. Jeltsch M, Kaipainen A, Joukov V, Meng X, Lakso M, Rauvala H, Swartz M, Fukumura D, Jain RK, Alitalo K. Hyperplasia of lymphatic vessels in VEGF-C transgenic mice. Science 1997; 276: 1423-5. 4. Dumont DJ, Jussila L, Taipale J, Lymboussaki A, Mustonen T, Pajusola K, Breitman M, Alitalo K
Cardiovascular failure in mouse embryos deficient in VEGF receptor-3. Science 1998; 282: 946-9.

5. Mattot V, Pourtier A, Soncin F, Vandenbunder B. La morphogenèse de l'arbre vasculaire : de la compréhension des mécanismes moléculaires aux perspectives thérapeutiques. Med Sci 1998; 14: 437-47. 6. Hiratsuka S, Minowa O, Kuno J, Noda T, Shibuya M. Flt-1 lacking the tyrosine kinase domain is sufficient for normal development and angiogenesis in mice. Proc Natl Acad Sci USA 1998; 95: 9349-54.

7. Bader BL, Rayburn H, Crowley D, Hynes RO. Extensive vasculogenesis, angiogenesis, and organogenesis precede lethality in mice lacking all alphav integrins. Cell 1998; 95 : 507-19.

\title{
BRÈVES
}

Un doigt de zinc pour les cheveux. L'alopécie universalis, caractérisée par une absence complète de poils et de cheveux est une maladie récessive autosomique extrêmement rare. Le gène en cause, découvert récemment $\left(\mathrm{m} / \mathrm{s} 1998, n^{\circ} 5, p .664\right)$, est l'homologue du gène murin hairless dont le phénotype a été décrit chez la souris depuis plus de 70 ans. La maladie fut d'abord étudiée dans une grande famille pakistanaise, elle a été retrouvée ensuite chez une famille de l'état d'Oman dans la péninsule arabique [1], puis chez des malades appartenant à une minorité ethnique, appelée les Irish Travellers, disséminés en Angleterre, en Irlande du Nord et en République d'Irlande [2]. Dans ce dernier pays, les Irish Travellers constituent $0,5 \%$ de la population totale et, avec une forte endogamie et une solide natalité, ils ont su préserver à travers les siècles leur culture et leur nomadisme [3]. L'examen clinique des malades de ce groupe montre qu'outre leur peau complètement glabre, ils ont, au niveau des genoux et des coudes, des lésions papuleuses et kystiques, comme dans une autre maladie rarissime: l'atrichie avec lésions papuleuses (MIM 209500). Étant donné la similitude de ces deux maladies cutanées, il est très probable qu'il s'agit d'une seule et même entité. On connaît la base moléculaire de la mutation murine hairless: une insertion du provirus de la leucémie murine dans l'exon 6 du gène. Les mutations humaines mises en évidence sont différentes dans les trois familles. La protéine déduite contient un seul domaine à doigt de zinc et doit agir comme facteur de transcription. Il a été récemment démontré que le gène hairless, qui est aussi exprimé dans le cer- veau, agit dans ce tissu comme un répresseur co-transcriptionnel, directement réglé par l'hormone thyroïdienne. En outre, chez la souris, on sait que le gène hairless s'exprime essentiellement dans l'épiderme et dans certaines structure du follicule. On peut donc supposer qu'il joue un rôle crucial dans le maintien de l'équilibre délicat entre prolifération, différenciation et apoptose dans le follicule pileux et dans l'épiderme interfolliculaire $\left(\mathrm{m} / \mathrm{s} \mathrm{1995,} n^{\circ} 9, p .1353\right)$. A quand le shampooing miracle pour les chauves?

[1. Gichon S, et al. Hum Mol Genet 1998; 7 : 1671-9.]

[2. Ahmad W, et al. Am J Hum Genet 1998; 63: 984-91.]

[3. Pavee point home page, http://homepages.iol.ie/ pavee/fs popul.htm]

\section{(9) \\ GROUPE DE RÉFLEXION SUR LA RECHERCHE CARDIOVASCULAIRE}

Sous le parrainage de l'INSERM et de la Société Française de Cardiologie

\author{
22-23 avril 1999 \\ Deauville-Palais des Congrès \\ Secrétariat scientifique (Résumés) \\ Pr. C. Thuillez, Service Pharmacologie, CHU - Rouen, 76031 Rouen Cedex \\ Fax : 0232889049 - e.mail : christianthuillez@chu.rouen.fr \\ Administration (Inscriptions et réservations hôtelières) \\ Deauville Organisation, Catherine Cutullic, BP 112 - 14800 Deauville \\ Tél : 0231985444 - Fax : 0231886576
}

